PROCEEDINGS OF THE

AMERICAN MATHEMATICAL SOCIETY

Volume 140, Number 7, July 2012, Pages 2501-2512

S 0002-9939(2011)11102-X

Article electronically published on November 18, 2011

\title{
ON $\omega$-CATEGORICAL GROUPS AND RINGS WITH NIP
}

\author{
KRZYSZTOF KRUPIŃSKI
}

(Communicated by Julia Knight)

\begin{abstract}
We prove that $\omega$-categorical rings with NIP are nilpotent-by-finite and that $\omega$-categorical groups with NIP and fsg are nilpotent-by-finite, too. We give an easy proof that each infinite, $\omega$-categorical $p$-group with NIP has an infinite, definable abelian subgroup. Assuming additionally that the group in question is characteristically simple and has a non-algebraic type which is generically stable over $\emptyset$, we show that the group is abelian.

Moreover, we prove that in any group with at least one strongly regular type all non-central elements are conjugated, and we conclude that assuming in addition $\omega$-categoricity, such a group must be abelian.
\end{abstract}

\section{INTRODUCTION}

Recall that a first-order structure $M$ in a countable language is said to be $\omega$ categorical if, up to isomorphism, $T h(M)$ has at most one model of cardinality $\aleph_{0}$. By Ryll-Nardzewski's theorem, this is equivalent to the condition that for every natural number $n$ there are only finitely many $n$-types over $\emptyset$. If $M$ is countable and $\omega$-categorical or if $M$ is a monster model (i.e. a model which is $\kappa$-saturated and strongly $\kappa$-homogeneous for a big cardinal $\kappa$ ), two finite tuples have the same type over $\emptyset$ iff they lie in the same orbit under the action of the automorphism group of $M$. Hence, for a countable $M$ or for $M$ being a monster model, $\omega$-categoricity means that for each natural number $n$ the automorphism group of $M$ has only finitely many orbits on $n$-tuples (which implies that $M$ is locally finite).

There is a long history of results describing the structure of $\omega$-categorical groups and rings. However, many questions in this area are still wide open. It follows easily that each countable, $\omega$-categorical group has a finite series of characteristic (i.e. invariant under the automorphism group) subgroups in which all successive quotients are characteristically simple groups (i.e. they do not have non-trivial, proper characteristic subgroups). On the other hand, Wilson (see [23, 1]) proved

Fact 0.1 . For each countably infinite, $\omega$-categorical, characteristically simple group $H$, one of the following holds.

(i) $H$ is an elementary abelian $p$-group for some prime $p$.

Received by the editors June 24, 2010 and, in revised form, February 25, 2011.

2010 Mathematics Subject Classification. Primary 03C45, 03C35, 20A15.

Key words and phrases. $\omega$-categorical group, $\omega$-categorical ring, non-independence property.

This research was supported by the Polish government grant N N201 545938 and by EPSRC grant EP/F009712/1.

(C)2011 American Mathematical Society Reverts to public domain 28 years from publication 
(ii) $H \cong B(F)$ or $H \cong B^{-1}(F)$ for some non-abelian, finite, simple group $F$, where $B(F)$ is the group of all continuous functions from the Cantor set $\mathcal{C}$ to $F$ and $B^{-}(F)$ is the subgroup of $B(F)$ consisting of the functions $f$ such that $f\left(x_{0}\right)=e$ for a fixed element $x_{0} \in \mathcal{C}$.

(iii) $H$ is a perfect $p$-group (perfect means that $H$ equals its commutator subgroup).

Moreover, it is conjectured that (iii) is not realized.

As to $\omega$-categorical rings in general, we know that their Jacobson radical is nilpotent (see [3, Lemma 1.3] and [5]). However, there are examples of infinite, $\omega$-categorical rings which are semisimple (i.e. with trivial Jacobson radical) and so not nilpotent-by-finite [3].

Interesting questions arise when one imposes additional model-theoretic restrictions (e.g. stability or simplicity) on our $\omega$-categorical group or ring. In the superstable [or, more generally, supersimple] $\omega$-categorical context, everything is clear: superstable groups are abelian-by-finite [4, 19] and supersimple groups are (finite central)-by-abelian-by-finite [7]; superstable rings are null-by-finite and supersimple rings are (finite null)-by-null-by-finite [11. In the stable [or, more generally, NSOP] situation, we only know that $\omega$-categorical groups are nilpotent-by-finite [4, 16, and $\omega$-categorical rings are nilpotent-by-finite [3, 13, too. It is an open question whether $\omega$-categorical stable groups are abelian-by-finite and whether $\omega$-categorical stable rings are null-by-finite.

Our motivating problem is to describe the structure of $\omega$-categorical groups and rings satisfying NIP (non-independence property; see Definition 1.4). Structures with NIP form a wide generalization of stable structures, covering o-minimal structures (e.g. the field of reals) and many other interesting examples (e.g. algebraically closed valued fields). Several important papers concerning NIP have been written in recent years, e.g. [9, 10, 20].

Reasonable conjectures on $\omega$-categorical groups and rings in the NIP context seem to be

Conjecture 0.2. Each $\omega$-categorical group with NIP is nilpotent-by-finite.

Conjecture 0.3. Each $\omega$-categorical ring with NIP is nilpotent-by-finite.

In this paper, we prove Conjecture 0.3. As to Conjecture 0.2 , we prove it under the additional assumption that the group has fsg (finitely satisfiable generics; see Definition (1.7). The fsg condition is an important notion which has been studied in recent years, e.g. in [9, 10, 6]. Recall that both stable groups and a certain wide class of groups definable in o-minimal structures have fsg and NIP.

One of the ingredients of our proof of Conjecture 0.2 is the result saying that each $\omega$-categorical, characteristically simple $p$-group with NIP and having a nonalgebraic, generically stable over $\emptyset$ type is abelian (so, under all these assumptions, (iii) of Fact 0.1 cannot happen). There is a short discussion on generic stability in Section 1. This notion (generalizing stability) has also been studied in recent literature, e.g in [21, 10, 17].

Shelah proved in [20] that if a group, which is a monster model, has NIP and an infinite abelian subgroup, then it has an infinite, definable, abelian subgroup. Since $\omega$-categorical groups are locally finite and each infinite, locally finite group has an infinite abelian subgroup [11, Corollary 2.5], one concludes that each infinite, $\omega$-categorical group with NIP has an infinite, definable, abelian subgroup. In this 
paper, we give an easy and direct proof of this result for $p$-groups (where $p$ is a prime number). It is worth mentioning that Plotkin [18] found examples (so-called extra special $p$-groups) of infinite, $\omega$-categorical $p$-groups with no infinite, definable, abelian subgroup.

At the end of the paper, we prove that in any group with at least one strongly regular type (see Definition 1.10) all non-central elements are conjugated, and we conclude that $\omega$-categorical groups with at least one strongly regular type are abelian.

\section{Preliminaries}

Recall that we say that a group $G$ is solvable-by-finite [nilpotent-by-finite or abelian-by-finite] if it has a finite index (normal) subgroup which is solvable [nilpotent or abelian, respectively].

It is standard (see e.g. [13, Remark 2.5]) that if $G$ is nilpotent-by-finite [abelianby-finite], then it has a definable normal subgroup of finite index which is nilpotent [abelian, respectively]. If $G$ is solvable-by-finite, it is not clear whether it has a definable, solvable subgroup of finite index (it has such a subgroup if we additionally assume either icc on centralizers for all definable quotients of definable subgroups [12, Remark 3.3] or $\omega$-categoricity).

Recall some basic notions from ring theory. In this paper, rings are associative, but they are not assumed to contain 1 or to be commutative. An element $r$ of a ring $R$ is nilpotent of nilexponent $n$ if $r^{n}=0$ and $n$ is the smallest number with this property. The ring is nil [of nilexponent $n$ ] if every element is nilpotent [of nilexponent $\leq n$ and there is an element of nilexponent $n]$. The ring is nilpotent of class $n$ if $r_{1} \cdots r_{n}=0$ for all $r_{1}, \ldots, r_{n} \in R$ and $n$ is the smallest number with this property. An element $r$ is null if $r R=R r=\{0\}$. The ring is null if all its elements are.

We say that a ring $R$ is nilpotent-by-finite [null-by-finite] if it has a finite index ideal (equivalently subring by [15]) which is nilpotent [null, respectively].

By virtue of [13, Remark 2.7], this ideal can be chosen definable.

The Jacobson radical of a ring $R$, denoted by $J(R)$, is the collection of all elements of $R$ satisfying the formula $\varphi(x)=\forall y \exists z(y x+z+z y x=0)$ (that is, it is the set of all elements which generate quasi-regular left ideals). Equivalently, $J(R)$ is the intersection of all the maximal regular left [or right] ideals, where a left ideal $I$ is said to be regular if there is $a \in R$ such that $x-x a \in I$ for all $x \in R$ (notice that for rings with 1 all ideals are regular). For any $\operatorname{ring} R, J(R)$ is a two-sided ideal. The ring $R$ is semisimple if $J(R)=\{0\}$. It is always the case that $R / J(R)$ is semisimple. For details on Jacobson radical see [8, Chapter 1].

Recall that a ring $R$ is a subdirect product of rings $R_{i}, i \in I$, if there is a monomorphism of $R$ into $\prod_{i \in I} R_{i}$ whose image projects onto each $R_{i}$. The following is [3, Corollary 1].

Fact 1.1. If $R$ is a semisimple, $\omega$-categorical ring, then $R$ is a subdirect product of complete matrix rings over finite fields. Moreover, only finitely many different matrix rings occur as subdirect factors.

By [3, Lemma 1.3] and [5] we have

Fact 1.2. If $R$ is an $\omega$-categorical ring, then $J(R)$ is nilpotent.

So, in order to prove that an $\omega$-categorical ring $R$ satisfying some extra assumptions is nilpotent-by-finite, it is enough to show that the semisimple ring $R / J(R)$ 
is finite (here Fact 1.1 may be very helpful). We will use this approach in the proof of Theorem 2.1.

We will also use [13, Theorem 3.15].

Fact 1.3. Suppose $G$ is a solvable, $\omega$-categorical group such that each ring interpretable in it is nilpotent-by-finite and each group $H$ definable in it has a definable connected component $H^{0}$ (i.e. the smallest definable subgroup of finite index). Then $G$ is nilpotent-by-finite.

Now, we recall the relevant notions from model theory. Let $T$ be a first-order theory. We work in a monster model $\mathfrak{C}$ of $T$.

Definition 1.4. We say that $T$ has the NIP if there is no formula $\varphi(x, y)$ and sequence $\left(a_{i}\right)_{i<\omega}$ such that for every $w \subseteq \omega$ there is $b_{w}$ such that $\models \varphi\left(a_{i}, b_{w}\right)$ iff $i \in w$.

The next fact is Theorem 1.0.5 of [22].

Fact 1.5. If $G$ is a group defined in a theory with NIP, then for each $\varphi$ there is some $N$ such that the intersection of any finite family of $\varphi$-definable subgroups of $G$ is an intersection of at most $N$ members of the family.

Assume $T$ has NIP and $G$ is a group $\emptyset$-definable in $\mathfrak{C}$. Shelah proved that then $G^{00}$ (the smallest type-definable subgroup of bounded index) exists (see 9 , Theorem 6.1]). Assume aditionally that $T$ is $\omega$-categorical. Since $G^{00}$ is $\emptyset$-invariant, it is $\emptyset$-definable, and so it has finite index and it is the connected component of $G$.

Let $p \in S(\mathfrak{C})$ be invariant over $A \subset \mathfrak{C}$. We say that $\left(a_{i}\right)_{i \in \omega}$ is a Morley sequence in $p$ over $A$ if $a_{n}=p \mid A a_{<n}$ for all $n$. It turns out that Morley sequences in $p$ over $A$ are indiscernible over $A$ and they have the same order type over $A$. If $\mathfrak{C}^{\prime} \succ \mathfrak{C}$ is a bigger monster model, then the generalized defining scheme of $p$ determines a unique $A$-invariant extension $\widetilde{p} \in S\left(\mathfrak{C}^{\prime}\right)$ of $p$ (by the generalized defining scheme of $p$ we mean a family of sets $\left\{p_{i}^{\varphi}: i \in I_{\varphi}\right\}$ (with $\varphi(x, y)$ ranging over all formulas without parameters) of complete types over $A$ such that $\varphi(x, c) \in p$ iff $\left.c \in \bigcup_{i \in I_{\varphi}} p_{i}^{\varphi}(\mathfrak{C})\right)$. By a Morley sequence in $p$ we mean a Morley sequence in $\widetilde{p}$ over $\mathfrak{C}$. Finally, $p^{(k)}$ (where $k \in \omega \cup\{\omega\}$ ) denotes the type over $\mathfrak{C}$ of a Morley sequence in $p$ of length $k$.

Recall that (correcting slightly the definition from [17]) a global type $p \in S(\mathfrak{C}$ ) is said to be generically stable if, for some small $A$, it is $A$-invariant and for each formula $\varphi(x, y)$ there is a natural number $n_{\varphi}$ such that for any Morley sequence $\left(a_{i}: i<\omega\right)$ in $p$ over $A$ and any $b$ from $\mathfrak{C}$ either at most $n_{\varphi} a_{i}$ 's satisfy $\varphi(x, b)$ or at most $n_{\varphi} a_{i}$ 's satisfy $\neg \varphi(x, b)$. Equivalently, $p$ is generically stable if, for some small $A$, it is $A$-invariant and for each Morley sequence $\left(a_{i}: i<\omega+\omega\right)$ in $p$ over $A$ and each formula $\varphi(x)$ (with parameters from $\mathfrak{C}$ ) the set $\left\{i: \models \varphi\left(a_{i}\right)\right\}$ is either finite or co-finite. In this definition, as a witness set $A$ one can take any (small) set over which $p$ is invariant. We will say that $p$ is generically stable over $A$ to express that $p$ is invariant over $A$ and generically stable. Assuming NIP, there are various equivalent definitions of generic-stability (see [10, Proposition 3.2]). For us, one of them will be particularly important.

Fact 1.6. Assume $T$ has NIP and $p \in S(\mathfrak{C})$ is $A$-invariant. Then, $p$ is generically stable iff every/some Morley sequence in $p$ over $A$ is an indiscernible set over $A$.

Now, we will briefly discuss fsg and generic stability. For more details on these and related notions see [21, 9, 10. 
Definition 1.7. Let $G$ be a group definable in $\mathfrak{C}$ by a formula $G(x)$.

(i) $G$ has fsg (finitely satisfiable generics) if there is a global type $p$ containing $G(x)$ and a model $M \prec \mathfrak{C}$, of cardinality less than the degree of saturation of $\mathfrak{C}$, such that for all $g, g p$ is finitely satisfiable in $M$.

(ii) $G$ is generically stable if $G$ has fsg and some global generic type of $G$ is generically stable (recall that a type $p$ is generic if for any formula $\varphi(x) \in p$ finitely many left translates of $\varphi(G)$ by elements of $G$ cover $G$ ).

We say that a group definable in a non-saturated model has one of the above properties if the group defined by the same formula in a monster model has it.

In general, generic stability of $G$ is a strictly stronger notion than fsg, but it is easy to check that these notions agree when $G^{00}$ is definable and $T$ has NIP. Namely, if $G$ has fsg and $G^{00}$ is definable, then $G^{00}$ also has fsg, and we can apply [10, Proposition 6.5]. In the $\omega$-categorical, NIP context, $G^{00}$ is definable, and thus we get

Remark 1.8. Assume $G$ is a group definable in an $\omega$-categorical structure with NIP. Then, it has fsg iff it is generically stable.

We leave the next remark as an easy exercise.

Remark 1.9. If a group $G$ definable in a model (of an arbitrary theory) has fsg, then the pure group $\langle G, \cdot\rangle$ also has fsg.

Finally, we recall the notion of a strongly regular type from [17.

Definition 1.10. Let $p(x) \in S(\mathfrak{C})$ be non-algebraic. We say that $p(x)$ is strongly regular if, for some small $A$, it is $A$-invariant and for all $B \supseteq A$ and $a$ from the sort of $x$ either $a \models p \mid B$ or $p|B \vdash p| B a$.

The geometric meaning of this notion is explained in [17, which we briefly recall now. Suppose $p \in S_{1}(\mathfrak{C})$ is non-algebraic and invariant over $\emptyset$. For a subset $C$ of $\mathfrak{C}$ we define $\operatorname{cl}_{p}(C)$ as $\{c \in \mathfrak{C}: c \not \models p \mid C\}$. Then, [17, Lemma 2] tells us that $p$ is strongly regular iff $c l_{p}$ is a closure operator (i.e. $c l_{p}\left(c l_{p}(C)\right)=c l_{p}(C)$ for all $C \subseteq \mathfrak{C}$ ). Assuming additionally that a Morley sequence in $p$ over $\emptyset$ is an indiscernible set, we get that $\left(\mathfrak{C}, c l_{p}\right)$ is a pregeometry.

\section{2. $\omega$-CATEGORICAL RINGS WITH NIP}

In this section, we prove Conjecture 0.3

Theorem 2.1. Each $\omega$-categorical ring with NIP is nilpotent-by-finite.

Proof. By Fact 1.2, everything boils down to showing that a semisimple, $\omega$-categorical ring $R$ with NIP is finite. Suppose for a contradiction that $R$ is infinite.

By $\omega$-categoricity, the two-sided ideals $R r R, r \in R$, are uniformly definable (because $\omega$-categoricity implies that there exists $K$ such that every element of any $R r R$ is the sum of at most $K$ elements of the form $r_{1} r r_{2}$ for $\left.r_{1}, r_{2} \in R \cup\{1\}\right)$. Thus, by NIP and Fact 1.5. there is $N \geq 1$ such that for all $n \in \omega$ and $r_{0}, \ldots, r_{n} \in R$, there are $i_{1}, \ldots, i_{N} \in\{0, \ldots, n\}$ such that $R r_{0} R \cap \cdots \cap R r_{n} R=R r_{i_{1}} R \cap \cdots \cap R r_{i_{N}} R$.

Fact 1.1 tells us that $R$ can be treated as a subring of the product $\prod_{i \in I} R_{i}$ of finite rings $R_{i}$ with identity, which projects onto each $R_{i}$, and where there are only 
finitely many pairwise distinct rings among the $R_{i}$ 's, $i \in I$. Let $\pi_{i}$ be the projection onto the $i$ th coordinate. For $i_{0}, \ldots, i_{n} \in I$ and $r_{j} \in R_{i_{j}}$, we introduce the set

$$
R_{i_{0}, \ldots, i_{n}}^{r_{0}, \ldots, r_{n}}=\left\{r \in R: \bigwedge_{j=0}^{n} \pi_{i_{j}}(r)=r_{j}\right\} .
$$

Using the assumption that $R$ is infinite and $R_{i}$ 's are finite, we see that for any $i_{0}, \ldots, i_{n} \in I$,

$$
R_{i_{0}, \ldots, i_{n}}^{0, \ldots, 0} \text { is an infinite two-sided ideal of } R \text {. }
$$

Claim 1. There are pairwise distinct $i_{0}, i_{1}, \cdots \in I$, non-nilpotent elements $r_{j} \in R_{i_{j}}$, and elements $\eta_{j} \in R$ such that for all $n \in \omega$,

$$
\eta_{n} \in R_{i_{0}, \ldots, i_{n}}^{0, \ldots, r_{n}} .
$$

Proof of Claim 1. Suppose $i_{0}, \ldots, i_{n}, r_{0}, \ldots, r_{n}$ and $\eta_{0}, \ldots, \eta_{n}$ have been chosen. Since $R$ is semisimple, it has no non-trivial nil left [or right or two-sided] ideals 8 , Lemma 1.2.2]. Thus, by $(*), R_{i_{0}, \ldots, i_{n}}^{0, \ldots, 0}$ contains a non-nilpotent element $\eta_{n+1}$. As there are only finitely many different $R_{i}$ 's and they are all finite, we can find $i_{n+1}$ different from $i_{0}, \ldots, i_{n}$ such that $r_{n+1}:=\pi_{i_{n+1}}\left(\eta_{n+1}\right) \in R_{i_{n+1}}$ is non-nilpotent.

Claim 2. There are natural numbers $n(0)<\cdots<n(N)$ such that the sets

$$
R_{i_{n(0)}, \ldots, i_{n(N)}}^{r_{n(0)}, 0, \ldots, 0}, R_{i_{n(0)}, \ldots, i_{n(N)}}^{0, r_{n(1)}, 0, \ldots, 0}, \ldots, R_{i_{n(0)}, \ldots, i_{n(N)}}^{0, \ldots, 0, r_{n(N)}}
$$

are non-empty.

Before proving Claim 2, let us notice that it leads to a contradiction. Choose $a_{0}, \ldots, a_{N}$ from $R_{i_{n(0)}, \ldots, i_{n(N)}}^{r_{n(0)}, \ldots, \ldots, 0}, \ldots, R_{i_{n(0)}, \ldots, i_{n(N)}}^{0, \ldots, 0, r_{n(N)}}$, respectively. Put $b_{k}=\sum_{l \neq k} a_{l}$ for $k=0, \ldots, N$. Then,

$$
\pi_{i_{n(j)}}\left[R b_{0} R \cap \cdots \cap R b_{N} R\right]=\{0\} \text { for } j=0, \ldots, N .
$$

On the other hand, $\prod_{k \neq j} b_{k} \in \bigcap_{k \neq j} R b_{k} R$ for $j=0, \ldots, N$. We also have that $\pi_{i_{n(j)}}\left[\prod_{k \neq j} b_{k}\right]=r_{n(j)}^{N} \neq 0$ as $r_{n(j)}$ is non-nilpotent. So,

$$
\pi_{i_{n(j)}}\left[\bigcap_{k \neq j} R b_{k} R\right] \neq\{0\} \text { for } j=0, \ldots, N .
$$

By $(* *)$ and $(* * *), R b_{0} R \cap \cdots \cap R b_{N} R \neq \bigcap_{k \neq j} R b_{k} R$ for all $j=0, \ldots, N$. This is a contradiction with the choice of $N$.

Proof of Claim 2. Let $c=\max _{i \in I}\left|R_{i}\right|$. Define recursively:

$$
\begin{aligned}
& L_{N}=c+1 \\
& L_{N-k}=c^{L_{N}+\cdots+L_{N-k+1}+1}+1 \text { for } k=1, \ldots, N-1 .
\end{aligned}
$$

Put $L_{0}=0$.

We will find

$$
\begin{aligned}
& n(0) \in I_{0}:=\left[L_{0}, L_{0}+L_{1}-1\right], \\
& n(1) \in I_{1}:=\left[L_{0}+L_{1}, L_{0}+L_{1}+L_{2}-1\right], \\
& \vdots \\
& n(N-1) \in I_{N-1}:=\left[L_{0}+\cdots+L_{N-1}, L_{0}+\cdots+L_{N}-1\right], \\
& n(N)=L_{0}+\cdots+L_{N}
\end{aligned}
$$


satisfying our demands. The essential of the definition of $L_{i}$ 's is the fact that the length of the interval $I_{N-1}$ is big enough, the length of the interval $I_{N-2}$ is big enough in comparison with the length of $I_{N-1}$, the length of $I_{N-3}$ is big enough in comparison with the length of $I_{N-2} \cup I_{N-1}$, and so on.

Consider any $k \in\{0, \ldots, N-1\}$. Suppose each natural number $\alpha$ from the closed interval $I_{N-k-1}=\left[L_{0}+\cdots+L_{N-k-1}, L_{0}+\cdots+L_{N-k}-1\right]$ has color

$$
\left(\pi_{i_{L_{0}+\cdots+L_{N-k}}}\left(\eta_{\alpha}\right), \ldots, \pi_{i_{L_{0}+\cdots+L_{N}}}\left(\eta_{\alpha}\right)\right) \in \prod_{j=L_{0}+\cdots+L_{N-k}}^{L_{0}+\cdots+L_{N}} R_{i_{j}} .
$$

In this way, the natural numbers from the interval $I_{N-k-1}$ have been colored with at most $c^{L_{N}+\cdots+L_{N-k+1}+1}=L_{N-k}-1$ colors (this formula also works for $k=0$, where we obviously have at most $c=L_{N}-1$ colors). Since there are $L_{N-k}$ such numbers, we can find two natural numbers $n(N-k-1)<n^{\prime}(N-k-1)$ from the interval $I_{N-k-1}$ with the same color. Put $a_{N-k-1}=\eta_{n(N-k-1)}-\eta_{n^{\prime}(N-k-1)}$. Then:

- Since $n(N-k-1)$ and $n^{\prime}(N-k-1)$ have the same color, we get that $\pi_{i_{j}}\left(a_{N-k-1}\right)=\pi_{i_{j}}\left(\eta_{n(N-k-1)}\right)-\pi_{i_{j}}\left(\eta_{n^{\prime}(N-k-1)}\right)=0$ for all $j \in\left[L_{0}+\cdots+\right.$ $\left.L_{N-k}, L_{0}+\cdots+L_{N}\right]$.

- By the choice of $\eta_{n}$ 's and the fact that $n^{\prime}(N-k-1)>n(N-k-1) \geq L_{0}+$ $\cdots+L_{N-k-1}$, we get $\pi_{i_{j}}\left(a_{N-k-1}\right)=\pi_{i_{j}}\left(\eta_{n(N-k-1)}\right)-\pi_{i_{j}}\left(\eta_{n^{\prime}(N-k-1)}\right)=$ $0-0=0$ for all $j \in\left[L_{0}, L_{0}+\cdots+L_{N-k-1}-1\right]$.

- By the choice of $\eta_{n}$ 's and the fact that $n(N-k-1)<n^{\prime}(N-k-1)$, we get $\pi_{i_{n(N-k-1)}}\left(a_{N-k-1}\right)=\pi_{i_{n(N-k-1)}}\left(\eta_{n(N-k-1)}\right)-\pi_{i_{n(N-k-1)}}\left(\eta_{n^{\prime}(N-k-1)}\right)=$ $r_{n(N-k-1)}-0=r_{n(N-k-1)}$.

Putting additionally $n(N)=L_{0}+\cdots+L_{N}$ and $a_{N}=\eta_{n(N)}$, we get

$$
a_{0} \in R_{i_{n(0)}, \ldots, i_{n(N)}}^{r_{n(0)}, 0, \ldots, 0}, a_{1} \in R_{i_{n(0)}, \ldots, i_{n(N)}}^{0, r_{n(1)}, 0, \ldots, 0}, \ldots, a_{N} \in R_{i_{n(0)}, \ldots, i_{n(N)}}^{0, \ldots, 0, r_{n(N)}} .
$$

So, the sequence $n(0)<\cdots<n(N)$ satsfies the conclusion of Claim 2 .

In this way, the proof of Theorem 2.1 has been completed.

\section{3. $\omega$-CATEGORICAL GROUPS WITH NIP}

In this section, we investigate the structure of $\omega$-categorical groups with NIP. First, we make some observations on characteristically simple groups in this context. Then, we prove Conjecture 0.2 under the additional assumptions of fsg. Finally, we show a variant of Conjecture 0.2 in which the NIP assumption is replaced by the existence of a strongly regular type.

As was mentioned in the introduction, each $\omega$-categorical group is locally finite, and so, if it is infinite, it has an infinite, abelian subgroup [11, Corollary 2.5]. Together with [20, Claim 4.3] this shows that an infinite, $\omega$-categorical group with NIP has an infinite, definable, abelian subgroup. We begin this section with a direct (avoiding [11, Corollary 2.5] and [20, Claim 4.3]) proof of this result for $p$-groups.

Proposition 3.1. Let $p$ be a prime number. Then every infinite, $\omega$-categorical p-group $G$ with NIP has an infinite, definable, abelian subgroup.

Proof. By NIP and $\omega$-categoricity, $G$ has a definable connected component. Replacing $G$ by its component, we can assume that $G$ is connected. If $Z(G)$ is infinite, we are done. Assume $Z(G)$ is finite. Then, $G / Z(G)$ is centerless, and we claim 
that it is enough to prove the proposition for $G / Z(G)$. Indeed, if $A$ is a definable subgroup of $G$ containing $Z(G)$ and such that $A / Z(G)$ is infinite and abelian, then the centralizer of every element of $A$ has finite index in $A$, and so the connected component of $A$ is an infinite, definable, abelian subgroup of $G$. Thus, we have reduced the situation to the case when $G$ is centerless.

For any $x_{0}, \ldots, x_{n} \in G,\left\langle x_{0}, \ldots, x_{n}\right\rangle$ is a finite $p$-group, and so $C\left(x_{0}\right) \cap \cdots \cap$ $C\left(x_{n}\right) \neq\{e\}$. Hence, if there were $x_{0}, \ldots, x_{n} \in G$ with $C\left(x_{0}\right) \cap \cdots \cap C\left(x_{n}\right)$ finite, we would find $x_{n+1}, \ldots, x_{m} \in G$ such that $Z(G)=C\left(x_{0}\right) \cap \cdots \cap C\left(x_{m}\right) \neq\{e\}$, a contradiction. So, we have proved the following:

Claim. For any $n \in \omega$ and $x_{0}, \ldots, x_{n} \in G$, the intersection $C\left(x_{0}\right) \cap \cdots \cap C\left(x_{n}\right)$ is infinite.

By the claim, we can choose a sequence $\left(x_{n}\right)_{n \in \omega}$ of pairwise distinct elements of $G$ such that $x_{n+1} \in C\left(x_{0}\right) \cap \cdots \cap C\left(x_{n}\right)$ for all $n \in \omega$. Put $G_{n}=C\left(x_{0}\right) \cap \cdots \cap C\left(x_{n}\right)$. Then, $x_{0}, \ldots, x_{n} \in Z\left(G_{n}\right)$. Hence, $\left|Z\left(G_{n}\right)\right|>n$.

On the other hand, by NIP and Fact 1.5, there is $N$ such that for any $y_{0}, \ldots, y_{n} \in$ $G$, there are $i_{1}, \ldots, i_{N} \in\{0, \ldots, n\}$ with $C\left(y_{0}\right) \cap \cdots \cap C\left(y_{n}\right)=C\left(y_{i_{1}}\right) \cap \cdots \cap C\left(y_{i_{N}}\right)$. Thus, $G_{n}=C\left(x_{i_{1}^{n}}\right) \cap \cdots \cap C\left(x_{i_{N}^{n}}\right)$ for some $i_{1}^{n}, \ldots, i_{N}^{n} \in\{0, \ldots, n\}$. Since by $\omega$ categoricity the set $\left\{t p\left(x_{i_{1}^{n}}, \ldots, x_{i_{N}^{n}}\right): n \in \omega\right\}$ is finite, there is $n \in \omega$ such that $Z\left(G_{n}\right)$ is infinite.

The next proposition uses notation from Fact 0.1 .

Proposition 3.2. For any non-abelian, finite, simple group $F$, neither $B(F)$ nor $B^{-}(F)$ has NIP.

Proof. Let $C_{0}, C_{1}, \ldots$ be disjoint clopen subsets of the Cantor set $\mathcal{C}$ not containing $x_{0}$. Choose $g \in F \backslash Z(F)$. Define a sequence $\left(f_{i}\right)_{i \in \omega}$ of elements of $B^{-}(F)$ by

$$
f_{i}(\eta)= \begin{cases}g & \text { if } \eta \in C_{i} \\ e & \text { if } \eta \notin C_{i}\end{cases}
$$

Now, suppose for a contradiction that $B(F)$ has NIP (the case when $B^{-}(F)$ has NIP is almost the same). Using NIP and Fact 1.5] and reordering $C_{i}$ 's if necessary, we can find $N$ such that $C_{B(F)}\left(f_{0}\right) \cap \cdots \cap C_{B(F)}\left(f_{N}\right)=C_{B(F)}\left(f_{0}\right) \cap \cdots \cap C_{B(F)}\left(f_{N-1}\right)$.

Take $h \in F \backslash C(g)$ and define $f \in B^{-}(F)$ by

$$
f(\eta)= \begin{cases}h & \text { if } \eta \in C_{N} \\ e & \text { if } \eta \notin C_{N}\end{cases}
$$

Then, we see that $f \in C_{B(F)}\left(f_{0}\right) \cap \cdots \cap C_{B(F)}\left(f_{N-1}\right) \backslash C_{B(F)}\left(f_{0}\right) \cap \cdots \cap C_{B(F)}\left(f_{N}\right)$, a contradiction.

Proposition 3.3. Let $p$ be a prime number. Let $M$ be an $\omega$-categorical structure with NIP. Assume $G$ is an infinite p-group which is $\emptyset$-definable in $M$ and characteristically simple in $M$ (i.e. $G$ does not have non-trivial, proper subgroups invariant under Aut $(M))$. Suppose that $G$ has a global generically stable over $\emptyset$ type $q$ different from the type of the neutral element. Then $G$ is abelian.

In particular, if $G$ is an infinite (pure), characteristically simple, $\omega$-categorical p-group with NIP possessing a global type $q$ which is generically stable over $\emptyset$ and which is not the type of the neutral element, then $G$ is abelian. 
Proof. Without loss of generality $M=\mathfrak{C}$ is a monster model (use the fact that in the monster model of an $\omega$-categorical theory invariant means $\emptyset$-definable).

Let $\left(a_{i}\right)_{i \in \omega}$ be a Morley sequence in $q$ over $\emptyset$. By NIP and Fact 1.5, there is $N$ such that for any $m, C\left(a_{0}\right) \cap \cdots \cap C\left(a_{m}\right)=C\left(a_{i_{1}}\right) \cap \cdots \cap C\left(a_{i_{N}}\right)$ for some $i_{1}, \ldots, i_{N} \in\{0, \ldots, m\}$. But, using Fact 1.6, $\left(a_{i}\right)_{i \in \omega}$ is an indiscernible set over $\emptyset$. This implies that for any $m \geq N-1$ and $0 \leq i_{1}<\cdots<i_{N} \leq m$, one has $C\left(a_{0}\right) \cap \cdots \cap C\left(a_{m}\right)=C\left(a_{i_{1}}\right) \cap \cdots \cap C\left(a_{i_{N}}\right)$.

Consider any $\left(b_{0}, b_{1}, \ldots\right) \models q^{(\omega)} \mid \emptyset$. Let $\left(c_{i}\right)_{i \in \omega}$ be a Morley sequence in $q$ over $a_{<\omega}, b_{<\omega}$. Then, the sequences $\left(a_{i}: i \in \omega\right) \frown\left(c_{i}: i \in \omega\right)$ and $\left(b_{i}: i \in \omega\right) \frown\left(c_{i}: i \in \omega\right)$ are indiscernible over $\emptyset$. Thus, by the last paragraph, $\bigcap_{i \in \omega} C\left(a_{i}\right)=C\left(c_{0}\right) \cap \cdots \cap$ $C\left(c_{N-1}\right)=\bigcap_{i \in \omega} C\left(b_{i}\right)$. But, $\left\langle c_{0}, \ldots, c_{N-1}\right\rangle \neq\{e\}$ is a finite $p$-group, which implies that $C\left(c_{0}\right) \cap \cdots \cap C\left(c_{N-1}\right) \neq\{e\}$. We conclude that $\bigcap_{i \in \omega} C\left(a_{i}\right)$ is a non-trivial, $\emptyset$-invariant in $\mathfrak{C}$ (so $\emptyset$-definable in $\mathfrak{C}$ ) subgroup of $G$. Since $G$ was characteristically simple in $\mathfrak{C}$, we get $\bigcap_{i \in \omega} C\left(a_{i}\right)=G$, which implies that $Z(G) \neq\{e\}$, and so $G=Z(G)$ once again by the characteristic simplicity of $G$.

Now, we are ready to prove Conjecture 0.2 under the fsg assumption.

Theorem 3.4. Each $\omega$-categorical group $G$ with NIP and fsg is nilpotent-by-finite.

Proof. We can assume that $G$ is an infinite, pure group which is a monster model. (In the $\omega$-categorical context, one usually considers pure groups, but here, even if we consider non-pure groups, Remark 1.9 allows us to replace the group by its reduct to the pure group structure.)

By NIP and $\omega$-categoricity, $G^{00}$ is $\emptyset$-definable, so it has fsg. Thus, using Remark 1.9, we can assume that $G=G^{00}$. Then, $G$ has a unique global generic type $q$ [6. Proposition 0.26], which must be $\emptyset$-invariant. So, by Remark [1.8, $q$ is generically stable over $\emptyset$, and it is also non-algebraic as a generic type of an infinite group.

By $\omega$-categoricty, there is a series $\{e\}=G_{0} \lesseqgtr G_{1} \lesseqgtr \cdots \lesseqgtr G_{n}=G$ of $\emptyset$-definable subgroups of $G$ of maximal possible (finite) length $n+1$. Since inner automorphisms belong to $\operatorname{Aut}(G)$, this series is normal; in particular, $G_{1} \unlhd G$. Moreover, $G_{1}$ is characteristically simple in $G$, which implies that $G_{1}$ is a characteristically simple group. The proof of the theorem will be by induction on $n$.

Consider the case $n=1$. Then, $G=G_{1}$ is an infinite, characteristically simple group. Proposition 3.2 eliminates the possibility that a countable elementary substructure of $G$ is as in point (ii) of Fact 0.1 . Proposition 3.3 together with our previous observation that $q$ is generically stable over $\emptyset$ and non-algebraic eliminates the possibility from point (iii) of Fact 0.1 Thus, $G$ must be abelian.

We turn to the induction step, where we assume that $n \geq 2$.

Claim. If $H$ is a non-trivial, $\emptyset$-definable subgroup of $G$, then $G / H$ is nilpotent.

Proof of Claim. As $G / H$ has fsg as a group interpretable in $G$, by Remark 1.9 and similar exercises, we conclude that the pure group $G / H$ also has fsg, NIP and it is $\omega$-categorical and connected. Moreover, we easily see that the maximal length of a sequence associated with $G / H$ is less than $n+1$ (for this notice that the preimage of a $\emptyset$-definable in the pure group $G / H$ subgroup of $G / H$ is a $\emptyset$-definable subgroup of $G$ ). Thus, by the induction hypothesis, $G / H$ is nilpotent-by-finite. So, it is nilpotent, because the Fitting subgroup of $G / H$ is a nilpotent subgroup of finite index which is $\emptyset$-definable in $G$ and so equal to $G / H$ by the connectedness of $G$. 
By the claim, if $Z(G)$ is non-trivial, then $G / Z(G)$ is nilpotent, and so $G$ is nilpotent. Thus, from now on we assume that $Z(G)=\{e\}$.

Once again by the claim, $G / G_{1}$ is nilpotent.

Let $\left(g_{i}\right)_{i \in \omega}$ be a Morley sequence in $q$ over $\emptyset$. Since $G / G_{1}$ is nilpotent, there is a minimal $k$ such that the iterated commutator $\left[g_{k-1},\left[g_{k-2}, \ldots,\left[g_{1}, g_{0}\right] \ldots\right]\right] \in G_{1}$. Since $g_{0}$ is generic over $\emptyset$ and $G / G_{1}$ is infinite, we see that $k \geq 2$. Define

$$
h_{i}=\left[g_{i k+k-1},\left[g_{i k+k-2}, \ldots,\left[g_{i k+1}, g_{i k}\right] \ldots\right]\right]
$$

for $i \in \omega$.

Let $\left(g_{i}^{\prime}\right)_{i \in \omega}$ be a Morley sequence in $q$ over $G$. Put

$$
h_{i}^{\prime}=\left[g_{i k+k-1}^{\prime},\left[g_{i k+k-2}^{\prime}, \ldots,\left[g_{i k+1}^{\prime}, g_{i k}^{\prime}\right] \ldots\right]\right]
$$

for $i \in \omega$. Since $t p\left(g_{0}^{\prime}, \ldots, g_{k-1}^{\prime} / G\right)=q^{(k)}$ is invariant over $\emptyset$, the type $r:=t p\left(h_{0}^{\prime} / G\right)$ is also invariant over $\emptyset$. Moreover, $\left(h_{i}\right)_{i \in \omega}$ is a Morley sequence in $r$ over $\emptyset$. By the generic stability of $q$ and Fact 1.6, the sequence $\left(g_{i}\right)_{i \in \omega}$ is an indiscernible set, and so $\left(h_{i}\right)_{i \in \omega}$ is an indiscernible set as well. We conclude that $r$ is generically stable over $\emptyset$.

We claim that $r$ is not the type of the neutral element. Otherwise $h_{0}=e$. Then, $g_{k-1} \in C(h)$, where $h=\left[g_{k-2}, \ldots,\left[g_{1}, g_{0}\right] \ldots\right] \neq e$ (from the minimality of $k$ ). Since $g_{k-1}$ is generic over $h$, we conclude that $[G: C(h)]<\omega$. But $G$ is connected, so $h \in Z(G)$, a contradiction.

Notice also that since $G$ is connected and $Z(G)=\{e\}, G_{1}$ is infinite.

Summarizing, $G_{1}$ is an infinite, $\emptyset$-definable subgroup of $G$ which is characteristically simple in $G$, and $r$ is a global type of $G_{1}$ which is generically stable over $\emptyset$ and which is not the type of the neutral element. So, by Proposition 3.3 together with Proposition 3.2 and Fact 0.1. $G_{1}$ is abelian. Hence, $G$ is solvable. By Theorem 2.1 and Fact 1.3. we conclude that $G$ is nilpotent-by-finite.

Dugald Macpherson told me an alternative ending of the above proof, i.e. an alternative proof of the fact that a solvable, $\omega$-categorical group with NIP is nilpotentby-finite. Namely, by [2, we know that each countable, solvable, $\omega$-categorical group which is not nilpotent-by-finite interprets the countable, atomless Boolean algebra. So, it remains to show that this algebra does not have NIP, which is an easy exercise.

Remark 3.5. If in Proposition 3.3 one was able to drop the assumption about the existence of a generically stable type $q$, then Conjecture 0.2 would be proved in its full generality.

Proof. This follows easily by induction on the maximal possible length of a series $\{e\}=G_{0} \lesseqgtr G_{1} \lesseqgtr \cdots \lesseqgtr G_{n}=G$ consisting of $\emptyset$-definable subgroups of $G$.

Now, we will drop the NIP and fsg assumption, and instead we will assume the existence of a strongly regular type. Recall the following question from [17].

Question 3.6. Suppose $G$ is a group with at least one strongly regular type. Does it imply that $G$ is abelian?

Proposition 3.7. If $G$ is any group with at least one strongly regular type, then all non-central elements of $G$ are conjugated. 
Proof. Taking an elementary extension of $G$, we can assume that there is a global type $p$ whose strong regularity is witnessed over $G$. Consider any non-central element $a \in G$. Take $b \models p \mid G$.

Notice that if $a^{b}=p \mid G$, then the formula defining the conjugacy class of $a$ belongs to $p \mid G$. Thus, all elements $a \in G$ for which $a^{b} \models p \mid G$ are in one conjugacy class. So, it remains to show that the assumption $a^{b} \not \models p \mid G$ leads to a contradiction.

This assumption and the strong regularity of $p$ over $G$ imply that $t p(b / G) \vdash$ $\operatorname{tp}\left(b / a^{b}, G\right)$. Thus, there is a formula $\varphi(x, y)$ (without parameters) and $g \in G^{n}$ such that $b \models \varphi(x, g)$ and $\models\left(\varphi(x, g) \rightarrow a^{x}=a^{b}\right)$. So, there is $c \in G$ such that $a^{c}=a^{b}$, and hence $b \in C(a) c$. This means that $p \mid G \vdash$ ' $x \in C(a) c$ '.

Consider two distinct realizations $g_{1}$ and $g_{2}$ of $p \mid G$ (they exist because $p$ is nonalgebraic).

Case $1(c \in C(a))$. Then, $p \mid G \vdash$ ' $x \in C(a)$ ', so $g_{1} \in C(a)$. Take $h \notin C(a)$. Then, $h g_{1} \notin C(a)$. Thus, by the strong regularity of $p$ over $G$, we conclude that $\operatorname{tp}\left(g_{1} / G, h, h g_{1}\right)=p \mid G, h, h g_{1}=t p\left(g_{2} / G, h, h g_{1}\right)$. But, the formula $x=h^{-1} h g_{1}$ belongs to $t p\left(g_{1} / G, h, h g_{1}\right)$ and does not belong to $t p\left(g_{2} / G, h, h g_{1}\right)$, a contradiction.

Case $2(c \notin C(a))$. Since $g_{1} \in C(a) c$, we have $g_{1} c^{-1} \in C(a)$, and so $g_{1} c^{-1} \notin$ $C(a) c$. Hence, by the strong regularity of $p$, we get $t p\left(g_{1} / G, g_{1} c^{-1}\right)=p \mid G, g_{1} c^{-1}=$ $t p\left(g_{2} / G, g_{1} c^{-1}\right)$. But, the formula $x=g_{1} c^{-1} c$ belongs to $t p\left(g_{1} / G, g_{1} c^{-1}\right)$ and does not belong to $t p\left(g_{2} / G, g_{1} c^{-1}\right)$, a contradiction.

Corollary 3.8. If $G$ is a group with at least one strongly regular type, then all non-central elements of $G$ have infinite order. In particular, an $\omega$-categorical group with at least one strongly regular type is abelian.

Proof. This is a standard argument. We can assume that $G \neq Z(G)$. Suppose for a contradiction that there is a non-central element of finite order. By the last proposition, $G / Z(G)$ has one non-trivial conjugacy class. So, all non-trivial elements of $G / Z(G)$ have the same order, which must be a prime number $p$. If $p=2$, then $G / Z(G)$ is abelian, so $[G: Z(G)] \leq 2$, which implies $G=Z(G)$, a contradiction. Now, we assume that $p$ is odd. Take a non-trivial $g \in G / Z(G)$. Then, there is $h \in G / Z(G)$ such that $h^{-1} g h=g^{-1}$. So, $g \in C\left(h^{2}\right) \backslash C(h)$. Finally we get

$$
C(h) \subsetneq C\left(h^{2}\right) \subsetneq \cdots \subsetneq C\left(h^{2^{p-2}}\right) \subsetneq C\left(h^{2^{p-1}}\right)=C(h),
$$

which is impossible.

\section{ACKNOWLEDGMENT}

The author is grateful to Dugald Macpherson for interesting discussions and suggestions.

\section{REFERENCES}

[1] A. Apps, On the structure of $\aleph_{0}$-categorical groups, Journal of Algebra 81, 320-339, 1983. MR700288 (84h:03079)

[2] R. Archer, D. Macpherson, Soluble, w-categorical groups, Math. Proc. Camb. Phil. Soc. 121, 219-227, 1997. MR1426520 (98c:03081)

[3] J. Baldwin, B. Rose, $\aleph_{0}$-categoricity and stability of rings, Journal of Algebra 45, 1-16, 1977. MR0439621 (55:12507)

[4] W. Baur, G. Cherlin, A. Macintyre, Totally categorical groups and rings, Journal of Algebra 57, 407-440, 1979. MR 533805 (80e:03034) 
[5] G. Cherlin, On $\aleph_{0}$-categorical nilrings. II, Journal of Symbolic Logic 45, 291-301, 1980. MR.569399 (81j:03053)

[6] C. Ealy, K. Krupiński, A. Pillay, Superrosy dependent groups having finitely satisfiable generics, Annals of Pure and Applied Logic 151, 1-21, 2008. MR2381504 (2009a:03041)

[7] D. Evans, F. Wagner, Supersimple $\omega$-categorical groups and theories, Journal of Symbolic Logic 65, 767-776, 2000. MR1771084 (2001f:03067)

[8] I. N. Herstein, Non-commutative Rings, Carus Mathematical Monographs, No. 15, Mathematical Association of America, 1968. MR0227205 (37:2790)

[9] E. Hrushovski, Y. Peterzil, A. Pillay, Groups, measures and the NIP, Journal of the AMS 21, 563-596, 2008. MR2373360 (2008k:03078)

[10] E. Hrushovski, A. Pillay, On NIP and invariant measures, Journal of the European Mathematical Society 13, 1005-1061, 2011. MR2800483

[11] O. Kegel, B.Wehrfritz, Locally Finite Groups, North-Holland, The Netherlands, 1973. MR0470081 (57:9848)

[12] K. Krupiński, Fields interpretable in rosy theories, Israel Journal of Mathematics 175, 421444, 2010. MR2607552

[13] K. Krupiński, On relationships between algebraic properties of groups and rings in some model-theoretic contexts, Journal of Symbolic Logic, accepted.

[14] K. Krupiński, F. Wagner, Small profinite groups and rings, Journal of Algebra 306, 494-506, 2006. MR2271348 (2008c:20052)

[15] J. Lewin, Subrings of finite index in finitely generated rings, Journal of Algebra 5, 84-88, 1967. MR0200297 (34:196)

[16] H. D. Macpherson, Absolutely ubiquitous structures and $\aleph_{0}$-categorical groups, Quart. J. Math. Oxford (2) 39, 483-500, 1988. MR975912 (90a:03043)

[17] A. Pillay, P. Tanović, Generic stability, regularity, and quasi-minimality, to appear in Categories and Models, a volume in honour of M. Makkai's 70th birthday.

[18] J. M. Plotkin, $Z F$ and locally finite groups, Zeitschrift für Math. Logik and Grundlagen der Mathematik 27, 375-379, 1981. MR626682 (83f:03044)

[19] B. Poizat, Stable groups, American Mathematical Society, Providence, 2001. MR.1827833 (2002a:03067)

[20] S. Shelah, Dependent first order theories, continued, Israel Journal of Mathematics 173, 1-60, 2009. MR 2570659

[21] A. Usvyatsov, On generically stable types in dependent theories, Journal of Symbolic Logic 74, 216-250, 2009. MR2499428 (2010b:03037)

[22] F. Wagner, Stable groups, London Math. Soc. Lecture Notes Series 240, Cambridge University Press, UK, 1997. MR1473226 (99g:20010)

[23] J. Wilson, The algebraic structure of $\aleph_{0}$-categorical groups, in Groups-St. Andrews 1981, C. M. Campbell, E. F. Robertson, eds., London Math. Soc. Lecture Notes 71, Cambridge, 345-358, 1981. MR679175 (84c:20006)

Instytut Matematyczny, Uniwersytet Wroceawski, Pl. GrunWaldzki 2/4, 50-384 WroCŁaW, Poland

E-mail address: kkrup@math.uni.wroc.pl 